\title{
SMN1 Duplications Are Associated With Progressive Muscular Atrophy, but Not With Multifocal Motor Neuropathy and Primary Lateral Sclerosis
}

Jeroen W. Bos, MD, Ewout J.N. Groen, PhD, Renske I. Wadman, MD, PhD, Chantall A.D. Curial, BS, Naomi N. Molleman, BS, Marinka Zegers, BS, Paul W.J. van Vught, PhD, Reinier Snetselaar, PhD,

Raymon Vijzelaar, PhD, W. Ludo van der Pol, MD, PhD, * and Leonard H. van den Berg, MD, PhD*

Neurol Genet 2021;7:e598. doi:10.1212/NXG.0000000000000598

\section{Abstract}

\section{Objective}

To assess the association between copy number $(\mathrm{CN})$ variation in the survival motor neuron $(S M N)$ locus and multifocal motor neuropathy (MMN), progressive muscular atrophy (PMA), and primary lateral sclerosis (PLS) susceptibility and to determine the association of SMN1 and SMN2 CN with MMN, PMA, and PLS disease course.

\section{Methods}

In this monocenter study, we used multiplex ligation-dependent probe amplification to determine SMN1 and SMN2 CN in Dutch patients with MMN, PMA, and PLS and controls. We stratified clinical parameters for SMN1 and SMN2 CN. We analyzed SMN1 and SMN2 exons $1-6$, intron 6, and exon $8 \mathrm{CN}$ to study the genetic architecture of SMN1 duplications.

\section{Results}

SMN1 and SMN2 CN were determined in 132 patients with MMN, 150 patients with PMA, 104 patients with PLS, and 956 control subjects. MMN and PLS were not associated with CN variation in SMN1 or SMN2. By contrast, patients with PMA more often than controls carried SMN1 duplications ( $\geq 3$ SMN1 copies, $12.0 \%$ vs $5.0 \%$, odds ratio 2.69 (1.43-4.91), p 0.0020). SMN1 and SMN2 CN status was not associated with MMN, PLS, or PMA disease course. In case of SMN1 exon 7 duplications, exons 1-6, exon 8, and introns 6 and 7 were also duplicated, suggesting full SMN1 duplications.

\section{Conclusions}

SMN1 duplications are associated with PMA, but not with PLS and MMN. SMN1 duplications in PMA are balanced duplications. The results of this study highlight the primary effect of altered SMN CN on lower motor neurons.
Correspondence

Prof. Dr. van den Berg Iberg@umcutrecht.nl 


\section{Glossary}

ALS = amyotrophic lateral sclerosis $\mathbf{C N}=$ copy number $\mathbf{C N V}=\mathrm{CN}$ variation $\mathbf{L M N}=$ lower motor neuron; $\mathbf{M L P A}=$ multiplex ligation-dependent probe amplification; $\mathbf{M M N}=$ multifocal motor neuropathy; $\mathbf{M R C}=$ Medical Research Council; OR = odds ratio; PAN = Prospective ALS Study in the Netherlands; PLS = primary lateral sclerosis; PMA = progressive muscular atrophy; SMA = spinal muscular atrophy; SMN = survival motor neuron; UMCU = University Medical Center Utrecht; UMN = upper motor neuron.

The survival motor neuron (SMN) locus, including SMN1 and the nearly identical SMN2, has gone through extensive duplication, deletion, and gene conversion events, causing variation in SMN1 and SMN2 copy number $(\mathrm{CN}) .{ }^{1}$ Heterogeneity at this locus has been associated with motor neuron disorders. Homozygous SMN1 deletions lead to lower motor neuron (LMN) degeneration in hereditary proximal spinal muscular atrophy (SMA), the severity of which is inversely correlated with SMN2 CN.,3 SMN1 duplications, by contrast, have been associated with amyotrophic lateral sclerosis (ALS), a neurodegenerative disease characterized by progressive loss of both upper MN (UMN) and LMN. ${ }^{4,5}$ No study has systematically investigated the association of other motor neuron disorders with SMN CN variation (CNV).

Multifocal motor neuropathy (MMN) is a rare, slowly progressive inflammatory neuropathy, characterized by asymmetrical distal muscle weakness, which responds to immunoglobulin treatment. ${ }^{6-8} \mathrm{MMN}$ is associated with specific HLA haplotypes, suggesting genetic susceptibility underlying disease pathogenesis. ${ }^{9}$ The selective motor neuron vulnerability seen in MMN is not fully explained. Progressive muscular atrophy (PMA) is characterized by progressive LMN degeneration, leading to muscular atrophy, muscle weakness, and, in some cases, premature death. Disease progression can be fast and reminiscent of ALS but is sometimes slow with patients surviving for decades. ${ }^{5,10}$ In contrast with the LMN vulnerability in PMA and MMN, primary lateral sclerosis (PLS) is characterized by progressive degeneration of UMNs alone. ${ }^{5}$

Here, we hypothesized that the SMN locus modifies susceptibility for a range of motor neuron disorders and determined SMN CNV in 3 large cohorts of Dutch MMN, PMA, and PLS patients.

\section{Methods}

\section{Study Population}

\section{Patients With MMN, Patients With PMA, Patients With PLS, and Controls}

All patients with MMN were diagnosed and enrolled at the outpatient clinic of the University Medical Center Utrecht (UMCU), a tertiary neuromuscular referral center and national center of expertise for MMN, ALS, and SMA. All patients fulfilled the most recent diagnostic criteria for definite, probable, or possible MMN. ${ }^{11}$ These criteria mostly rely on the combination of a typical clinical phenotype combined with conduction block found on nerve conduction studies or, in the absence of conduction block, on the basis of abnormal ancillary investigations and/or a response to treatment with immunoglobulins. All patients with MMN were Dutch.

Patients with PMA and PLS were enrolled through the Prospective ALS Study in the Netherlands (PAN), a Dutch population-based prospective case-control study. ${ }^{12}$ All patients with PMA suffered from a progressive LMN loss without signs or symptoms consistent with UMN involvement, that is, no pseudobulbar affect, increased jaw jerk, muscle hypertonia, pathologic deep tendon reflexes, or pathologic signs including extensor plantar (Babinski) reflex or ankle clonus. Patients with PLS showed selective UMN loss, that is, pseudobulbar dysarthria, pseudobulbar affect, hypertonia, pathologic deep tendon reflexes, or pathologic signs including extensor plantar (Babinski) reflexes or clonus. None of the patients with PLS showed signs or symptoms of LMN degeneration, that is, no atrophy, fasciculations, or diminished or absent deep tendon reflexes.

Dutch population-based controls were also enrolled through the PAN. ${ }^{12}$ Controls enrolled between January 2012 and August 2018 were included in this study. None of the controls were diagnosed with MND after inclusion in this study.

\section{Clinical Data}

For patients with MMN, PMA, and PLS, clinical data were drawn from the MMN or PAN database. ${ }^{12,13}$ When needed, these data were supplemented with data from UMCU patient files.

For all patients, recorded data included sex, age at onset, diagnostic delay, site of onset, response to intravenous immunoglobulin treatment, presence of anti-GM1 IgM antibodies, and muscle strength testing on the first and last visit to the outpatient clinic. Results of nerve conduction studies were used to assess the presence of conduction block in patients with MMN. Survival data from patients with PMA and PLS were obtained by checking the last date the patient was known to be alive in the municipal population register (updated at quarterly intervals).

Onset of disease was defined as the first complaint of muscle weakness. Diagnostic delay was defined as the time between 
the onset of muscle weakness and diagnosis. Anti-GM1 IgM antibody testing was performed using standardized ELISA. ${ }^{14}$ Results of muscle strength testing on the first and last visit to the outpatient clinic were quantified on the 6-point Medical Research Council (MRC) scale. This scale ranges from 0 (no muscle contraction) to 5 (normal muscle strength against resistance). We documented the MRC scores for left and right shoulder abduction, elbow flexion and extension, wrist flexion and extension, finger flexion and extension, finger spreading, hip flexion, knee flexion and extension, and foot dorsal and plantar flexion. An MRC sum score was calculated by the summation of the MRC scores of all tested muscle groups (range 0-130). As an outcome measure in patients with MMN who were untreated at their first visit, a $\triangle \mathrm{MRC}$ sum score/ month was calculated by subtracting the MRC sum score at the first from that at the last visit, divided by the follow-up time.

In patients with $\mathrm{MMN}$, nerve conduction studies were performed as described previously. ${ }^{9,13}$ In short, motor and sensory function were tested bilaterally in the median, ulnar, radial, musculocutaneous, peroneal, and tibial nerves. In nerves with a distal CMAP of $>1 \mathrm{mV}$, the presence of conduction blocks was assessed, where a definite block was defined as a CMAP reduction of at least $50 \%$ and a probable block as a reduction of $30 \%-50 \%$.

Survival was defined as the time between the onset of muscle weakness and death or last date known alive.

\section{DNA Samples}

Genomic DNA was extracted from whole blood using standard DNA isolation methods. Samples from controls, patients with PMA, and patients with PLS were obtained upon participation in the PAN. Samples from patients with MMN were obtained during the Dutch national cross-sectional studies on MMN performed in 2007 and 2015.

\section{SMN CNV Analysis}

We used multiplex ligation-dependent probe amplification (MLPA) to assess CNV in the SMN locus. We used the SALSA MLPA P021-B1 SMA probe mix (MRC Holland, Amsterdam, The Netherlands). All analyses were performed as described previously. ${ }^{2}$

SMN1 and SMN2 exon 7 specific probes were used to determine the $S M N 1$ and $S M N 2 \mathrm{CN}$. The $S M N 1 / 2 \Delta 7-8$ gene variant $\mathrm{CN}$ was determined by subtracting the median $\mathrm{CN}$ of the 7 probes targeting $S M N 1$ and $S M N 2$ intron 6, as well as exons 7 and 8 from the median $\mathrm{CN}$ of the 10 probes targeting $S M N 1$ and $S M N 2$ exons 1-6. For example, when the median $\mathrm{CN}$ of the intron 6-exon 8 region of $S M N 1$ and $S M N 2$ was 3 and the median $\mathrm{CN}$ of $S M N 1$ and $S M N 2$ exons 1-6 was 4, this indicated the presence of 1 copy of the $S M N 1 / 2 \Delta 7-8$ gene variant. ${ }^{15}$

\section{Statistical Analysis}

We used R (version 3.6.1) to perform statistical analyses. $S M N 1, S M N 2$, and $S M N 1 / 2 \Delta 7-8 \mathrm{CN}$ in patients with MMN,
PMA, and PLS were separately compared with controls using a $\chi^{2}$ or Fisher exact test, as appropriate. Odds ratios and $95 \%$ confidence intervals were calculated.

Patients were stratified by the SMN1 CN ( 1,2 , or $\geq 3$ copies). To assess possible $S M N 2$ disease modification, clinical parameters within each $S M N 1$ group were stratified by the SMN2 CN. We compared continuous variables using ANOVA or Kruskal-Wallis testing, as appropriate. In case of a $p$ value of $<0.05$, a pairwise $t$ test or Wilcoxon signed-rank test was performed. We used a prerequisite of at least 5 observations per group for pairwise comparisons.

Survival analyses within the PMA and PLS groups were performed using the "Survminer" package. Kaplan-Meier curves were drawn, stratified by the SMN1 CN. At least 5 observations per stratum were required for survival analyses. The assumption of proportional hazard was tested, and hazard ratios with $95 \%$ confidence intervals were calculated. To assess possible $S M N 2$ disease modification, each $S M N 1$ group was stratified by the $S M N 2 \mathrm{CN}$, and the same survival analyses were performed.

$p$-Value adjustment using the Bonferroni method was applied in case of multiple testing. A corrected $p$ value of $<0.05$ was considered statistically significant.

\section{Standard Protocol Approvals, Registrations, and Patient Consents}

The locally appointed ethics committee of the University Medical Center Utrecht gave approval for this study (METC Utrecht, METC.NL.041). All included patients gave written informed consent before inclusion in this study.

\section{Data Availability}

The data that support the findings in this study will be available on reasonable request from the corresponding author.

\section{Results}

\section{Study Population}

We included 137 patients with MMN, 159 patients with PMA, 105 patients with PLS, and 981 controls. We obtained results from 132 patients with MMN (96.4\%), 150 patients with PMA (94.3\%), 104 patients with PLS (99\%), and 956 controls (97.4\%). Baseline characteristics are shown in table 1. Sex, age at onset, diagnostic delay, and site of onset were recorded in all patients. Clinical parameters of patients with $M M N$, that is, the first visit MRC sum score and the number of affected limbs at the first visit, are shown for patients who were treatment naive at their first visit $(\mathrm{n}=102)$. Clinical parameters in the PMA group were recorded for all included patients. At the end of this study, $55 \%$ and $36 \%$ of patients with PMA and patients with PLS had deceased, of whom $47 \%$ and $13 \%$, respectively, had died within 4 years after disease onset. 
Table 1 Baseline Characteristics of Patients With MMN, PMA, and PLS

\begin{tabular}{|c|c|c|c|c|}
\hline & MMN $(n=132)$ & PMA $(n=150)$ & PLS $(n=104)$ & Controls $(n=956)$ \\
\hline Male sex (n, \%) & $102(77)$ & $119(79)$ & $57(55)$ & $592(62)$ \\
\hline Age $^{a}$ & $42(15)$ & $62(15)$ & $58(16)$ & $66(9)$ \\
\hline Delay in $\mathbf{m o}^{a}$ & $42(62)$ & $17(18)$ & $34(43)$ & \\
\hline \multicolumn{5}{|l|}{ Site of onset $(n, \%)$} \\
\hline Bulbar & $0(0)$ & $1(1)$ & $25(24)$ & \\
\hline Spinal & $132(100)$ & $146(97)$ & $79(76)$ & \\
\hline Thoracic/respiratory & $0(0)$ & $3(2)$ & $0(0)$ & \\
\hline \multicolumn{5}{|l|}{ EFNS diagnosis $(n, \%)$} \\
\hline Definite MMN & $94(73)$ & NA & NA & \\
\hline Probable MMN & $24(19)$ & NA & NA & \\
\hline Possible MMN & $10(8)$ & NA & NA & \\
\hline Anti-GM1 IgM antibodies (n/N, \%) & $75 / 116(65)$ & $2 / 18(11)$ & $0 / 0(0)$ & \\
\hline IVIg response (n/N, \%) & $118 / 126(94)$ & $0 / 2(0)$ & $0 / 0(0)$ & \\
\hline IVIg treatment at the first visit (n/N, \%) & $27 / 129(21)$ & $0 / 150(0)$ & $0 / 0(0)$ & \\
\hline Disease duration at the first visit in $\mathrm{mo}^{\mathrm{b}}$ & $31(3-433)$ & $13(2-485)$ & $28(1-325)$ & \\
\hline MRC sum score at the first visit ${ }^{b}$ & $122(85-130)$ & $122(80-129)$ & $130(105-130)$ & \\
\hline \multicolumn{5}{|l|}{ Limbs affected at the first visit } \\
\hline $1(n, \%)$ & $35(34)$ & $32(21)$ & $13 / 98(13)$ & \\
\hline $4(n, \%)$ & $24(24)$ & $48(32)$ & 10/98 (10) & \\
\hline Riluzole treatment $(\mathrm{n}, \%)$ & $0(0)$ & $130(87)$ & $89(86)$ & \\
\hline Follow-up data $(n, \%)$ & $120(91)$ & $50(33)$ & $59(57)$ & \\
\hline Follow-up duration in $\mathrm{mo}^{\mathrm{a}}$ & $101(134)$ & $22(28)$ & $31(31)$ & \\
\hline C9ORF72 repeat expansions ( $n, \%)$ & NA & $2 / 148(1.4)$ & $1 / 104(1)$ & \\
\hline Overall mortality & NA & $83(55)$ & $37(36)$ & \\
\hline Death within 4 years of onset ( $n, \%)$ & NA & $39(47)$ & $5(14)$ & \\
\hline \multicolumn{5}{|c|}{$\begin{array}{l}\text { Abbreviations: IVIg = intravenous immunoglobulins; MRC = Medical Research Council; MMN = multifocal motor neuropathy; NA = not applicable; PLS = } \\
\text { primary lateral sclerosis; PMA = progressive muscular atrophy. } \\
\text { Clinical parameters at the first visit are shown for treatment-naive MMN patients alone }(n=102) . \\
\text { a Values displayed as median (IQR). } \\
\text { b Values displayed as median (range). }\end{array}$} \\
\hline
\end{tabular}

\section{CNV Analysis}

SMN1, SMN2, and SMN1/2 7 -8 CN are shown in table 2. In controls, we observed 48 SMN1 duplications, of which 1 person had 4 and 1 person had 5 copies. All 11 patients with MMN with SMN1 duplications had 3 copies. Eighteen patients with PMA had SMN1 duplications, of which 3 had 4 copies. Seven patients with PLS had a single SMN1 duplication.

Compared with controls, SMN1 duplications were found more frequently in patients with PMA (12.0\% vs $5.0 \%$, odds ratio [OR] 2.69 (1.43-4.91), p 0.0020), but not in patients with MMN and PLS. This association is further exemplified by the fact that also double SMN1 duplications (i.e., at least 4 SMN1 copies) were found more frequently in patients with PMA (2\% vs $0.2 \%$, OR 11.0 [1.25-133.56], p 0.015). No associations with SMN2 CNV were found, nor with the presence of the $S M N 1 / 2 \Delta 7-8$ gene variant. The average total SMN CN was comparable between the patient groups and controls, possibly indicating an SMN2 deletion event accompanying SMN1 duplications.

A detailed overview of the SMN1:SMN2 configuration in controls and patients with PMA is included in supplementary table e-1, links.lww.com/NXG/A429. 
Table 2 SMN1, SMN2, and SMN1/2A7-8 Copy Numbers in Controls $(\mathrm{n}=956)$ and Patients With MMN ( $\mathrm{n}=132)$, PMA $(n=150)$, and PLS $(n=104)$

\begin{tabular}{|c|c|c|c|c|c|c|c|c|c|c|}
\hline & Controls & MMN & $p$ Value & OR $(95 \% \mathrm{Cl})$ & PMA & $p$ Value & OR $(95 \% \mathrm{Cl})$ & PLS & $p$ Value & OR $(95 \% \mathrm{Cl})$ \\
\hline \multicolumn{11}{|l|}{$S M N 1^{a}$} \\
\hline 1 & $20(2.1)$ & $4(3.0)$ & 0.52 & $1.46(0.36-4.46)$ & $4(2.7)$ & 0.56 & $1.28(0.31-3.90)$ & $1(1.0)$ & 0.71 & $0.45(0.01-2.90)$ \\
\hline 2 & $888(92.9)$ & $117(88.6)$ & 0.12 & $0.59(0.32-1.16)$ & $128(85.3)$ & $0.0028^{c}$ & $0.45(0.26-0.79)$ & $96(92.3)$ & 0.99 & $0.92(0.42-2.28)$ \\
\hline$\geq 3$ & $48(5.0)$ & $11(8.3)$ & 0.17 & $1.72(0.78-3.47)$ & $18(12.0)$ & $0.0020^{c}$ & $2.69(1.43-4.91)$ & $7(6.7)$ & 0.48 & $1.36(0.50-3.14)$ \\
\hline \multicolumn{11}{|l|}{$S M N 2^{\mathrm{a}}$} \\
\hline 0 & $78(8.2)$ & $11(8.3)$ & 1.00 & $1.02(0.48-2.00)$ & $11(7.3)$ & 0.85 & $0.89(0.42-1.74)$ & $9(8.7)$ & 0.85 & $1.07(0.46-2.22)$ \\
\hline 1 & 401 (41.9) & $56(42.4)$ & 0.99 & $1.02(0.69-1.50)$ & $74(49.3)$ & 0.11 & $1.34(0.94-1.93)$ & 35 (33.7) & 0.13 & $0.70(0.44-1.09)$ \\
\hline 2 & $451(47.2)$ & $59(44.7)$ & 0.66 & $0.91(0.62-1.33)$ & $60(40.0)$ & 0.12 & $0.75(0.52-1.07)$ & 54 (51.9) & 0.41 & $1.21(0.79-1.85)$ \\
\hline 3 & $24(2.5)$ & $6(4.5)$ & 0.29 & $185(0.61-4.76)$ & $5(3.3)$ & 0.58 & $1.34(0.29-3.66)$ & $4(3.8)$ & 0.35 & $1.55(0.38-4.65)$ \\
\hline 4 & $2(0.2)$ & - & - & - & - & - & - & $2(1.9)$ & 0.05 & $9.31(0.67-130)$ \\
\hline \multicolumn{11}{|l|}{$S M N 1 / 2 \Delta 7-8^{a}$} \\
\hline 0 & $718(75.1)$ & $102(77.3)$ & 0.66 & $1.13(0.72-1.80)$ & $104(69.3)$ & 0.16 & $0.74(0.51-1.12)$ & $83(79.8)$ & 0.34 & $1.31(0.78-2.27)$ \\
\hline 1 & $230(24.1)$ & $27(20.5)$ & 0.42 & $0.81(0.50-1.29)$ & $45(30.0)$ & 0.14 & $0.35(0.90-2.00)$ & $20(19.2)$ & 0.33 & $0.75(0.43-1.27)$ \\
\hline 2 & $8(0.8)$ & $3(2.3)$ & 0.14 & $2.75(0.50-11.6)$ & $1(0.7)$ & 1.00 & $0.80(0.02-6.00)$ & $1(1.0)$ & 0.61 & $1.15(0.03-8.72)$ \\
\hline \multicolumn{11}{|l|}{ Mean $\mathrm{CN}^{\mathrm{b}}$} \\
\hline SMN1/SMN2 & $3.48(0.72)$ & $3.51(0.78)$ & 0.68 & - & $3.50(0.76)$ & 0.67 & - & $3.62(0.89)$ & 0.11 & - \\
\hline $\begin{array}{l}S M N 1 / S M N 2 I \\
S M N 1 / 2 \triangle 7-8\end{array}$ & $3.74(0.64)$ & $3.76(0.67)$ & 0.73 & - & $3.82(0.75)$ & 0.15 & - & $3.84(0.75)$ & 0.19 & - \\
\hline
\end{tabular}

Abbreviations: $\mathrm{CN}$ = copy number; $\mathrm{MMN}$ = multifocal motor neuropathy; $\mathrm{OR}$ = odds ratio; PLS = primary lateral sclerosis; PMA = progressive muscular atrophy; $\mathrm{SMN}=$ survival motor neuron.

a Values displayed as $\mathrm{n}(\%)$.

b Values displayed as mean (SD).

'Statistically significant after Bonferroni $p$-value adjustment. Uncorrected $p$ values are shown.

\section{Clinical Correlation}

In all patient groups, age at onset and MRC sum score at the first visit were compared by $S M N 1$ group (1,2, and $\geq 3$ copies). Because of low numbers in groups with an $S M N 1$ deletion and duplication, the modifying potential of the SMN2 CN was analyzed in the group with 2 SMN1 copies alone. Results are shown in figure 1.

We did not find a positive association between SMN1 and SMN2 CN and median age at onset or median MRC sum scores at the first visit in patients with MMN, PMA, or PLS. In the MMN group, SMN1 CN and SMN2 CN within patients carrying 2 SMN1 copies did not alter the rate of progression as expressed by $\triangle \mathrm{MRC}$ sum score/month ( $p$ values 0.36 and 0.52 , respectively, data not shown).

SMN1 duplications were not found more frequently in patients with PMA and a survival longer than 48 months (Fisher's exact test $p$ 0.30, data not shown). Kaplan-Meier curves were drawn for all patients with PMA and PLS carrying 2 SMN1 copies and SMN1 duplications. Because of the low number of patients with an SMN1 deletion, this group was excluded from survival analysis. Kaplan-Meier curves are shown in figure 2. Cox proportional hazard assumption was met in all analyses $(p>0.05)$. Although, compared with patients with PMA carrying 2 SMN1 copies, survival in patients with PMA with SMN1 duplications was lower, this was not statistically significant. SMN2 CN did not affect survival in patients with PMA or PLS carrying 2 SMN1 copies.

\section{SMN1 Duplication Analysis}

To gain further insight in the genetic architecture of the SMN locus in case of SMN1 duplications, we further analyzed patients with PMA and controls with 3 SMN1 copies ( $n=15$ and 46 , respectively). Results are shown in table 3.

In all subjects carrying $1 S M N 1$ duplication as expressed by the $S M N 1$ exon $7 \mathrm{CN}$, the $S M N 1$ exon $8 \mathrm{CN}$ matched the SMN1 exon $7 \mathrm{CN}$. The same accounts for SMN2 exon 7 and 8 CN. In 36 controls (78\%) and 14 patients with PMA (93\%), the SMN1 and SMN2 exons 1-6 and intron $6 \mathrm{CN}$ matched the combined $S M N 1$ and $S M N 2$ exon $7 \mathrm{CN}$, suggesting a balanced and full duplication of SMN1. In 10 controls (22\%) and 1 patient with PMA (7\%), one copy of the $S M N 1 / 2 \Delta 7-8$ gene variant was found. Besides carrying 1 extra copy of 

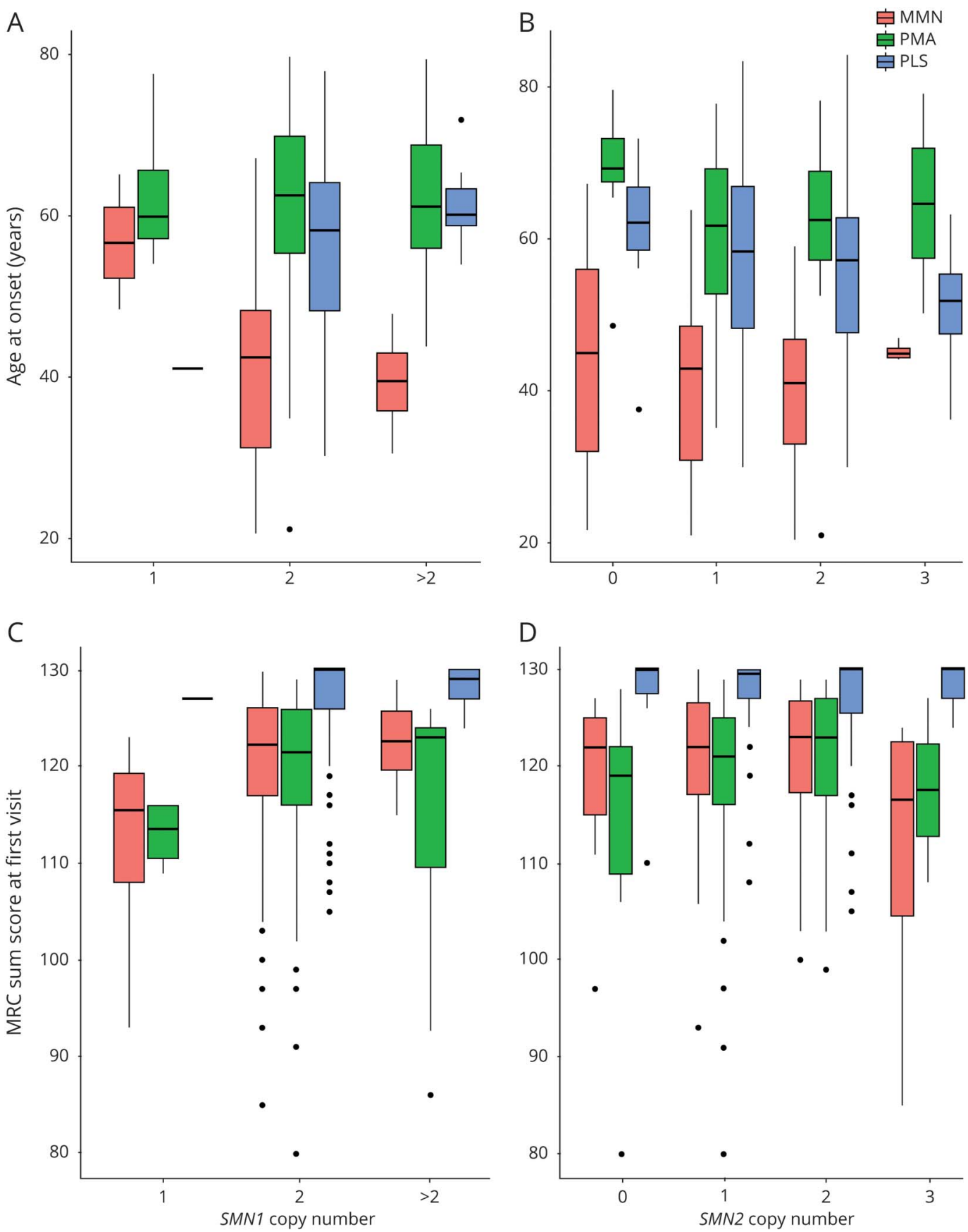

Boxplots showing median age at onset in years and median MRC sum score at the first visit in patients with MMN, PMA, and PLS. A) Age at onset in years by the SMN1 copy number. B) Age at onset by the SMN2 copy number in patients carrying 2 SMN1 copies. C) MRC sum score at the first visit by the SMN1 copy number. D) MRC sum score at the first visit by the SMN2 copy number in patients carrying 2 SMN1 copies. No association between the clinical parameters and SMN1 or $S M N 2$ copy number status was found in either disease group (all $p$ values $>0.05$ ). $\mathrm{MMN}=$ multifocal motor neuropathy; $\mathrm{MRC}=$ Medical Research Council; $\mathrm{PLS}=$ primary lateral sclerosis; PMA = progressive muscular atrophy; SMN = survival motor neuron.

SMN1 and SMN2 exons 1-6 and intron 6, these subjects all have a probable full SMN1 gene duplication.

Regarding the SMN1:SMN2 genetic architecture, there was a trend toward patients with PMA having a higher SMN2 and thus lower $S M N 1 / 2 \Delta 7-8 \mathrm{CN}$ when compared with controls, although this was not statistically significant (Fisher's exact test $p 0.64$ and 0.26 , respectively). Telomeric NAIP exon 5 duplications were found in
45 of 61 SMN1 duplications (73\%), which did not differ between patients with PMA and controls (Fisher's exact test $p$ 0.51).

\section{Discussion}

In this study, we investigated the possible association between $\mathrm{CNV}$ in the SMN locus and the motor neuron disorders 


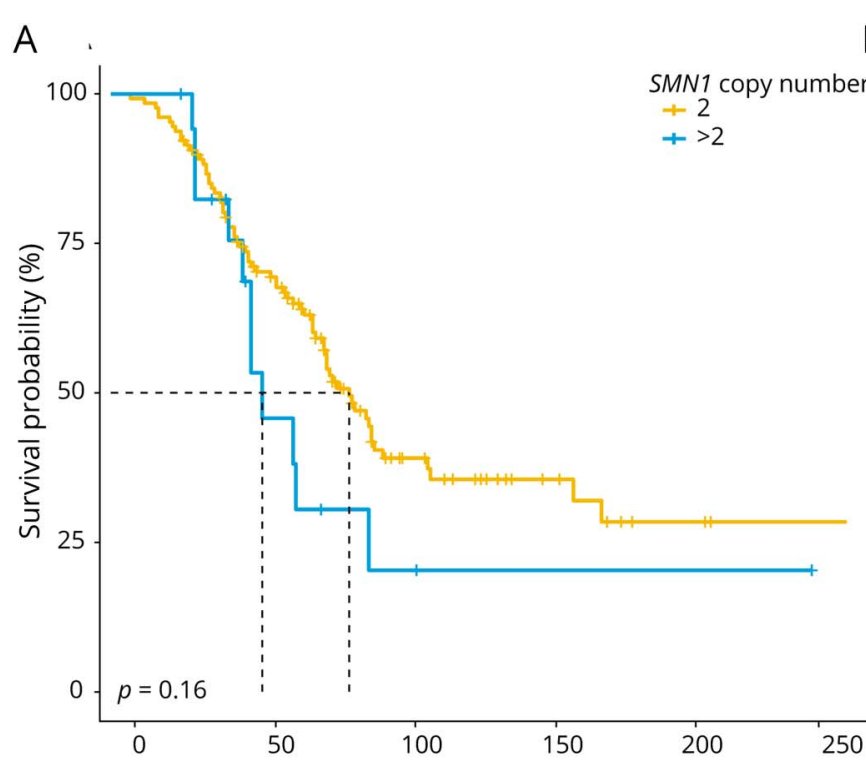

\section{B}

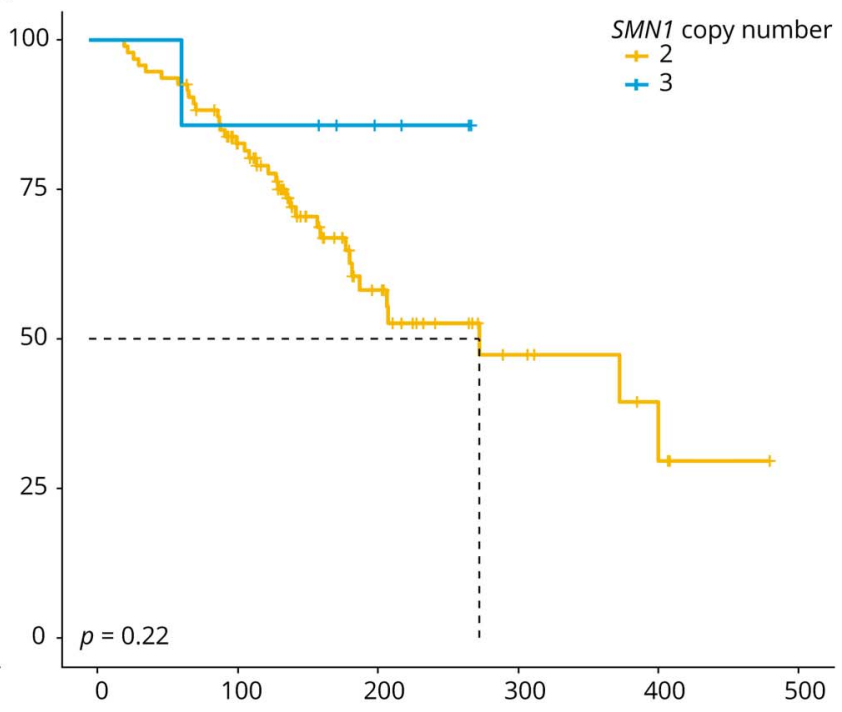

C
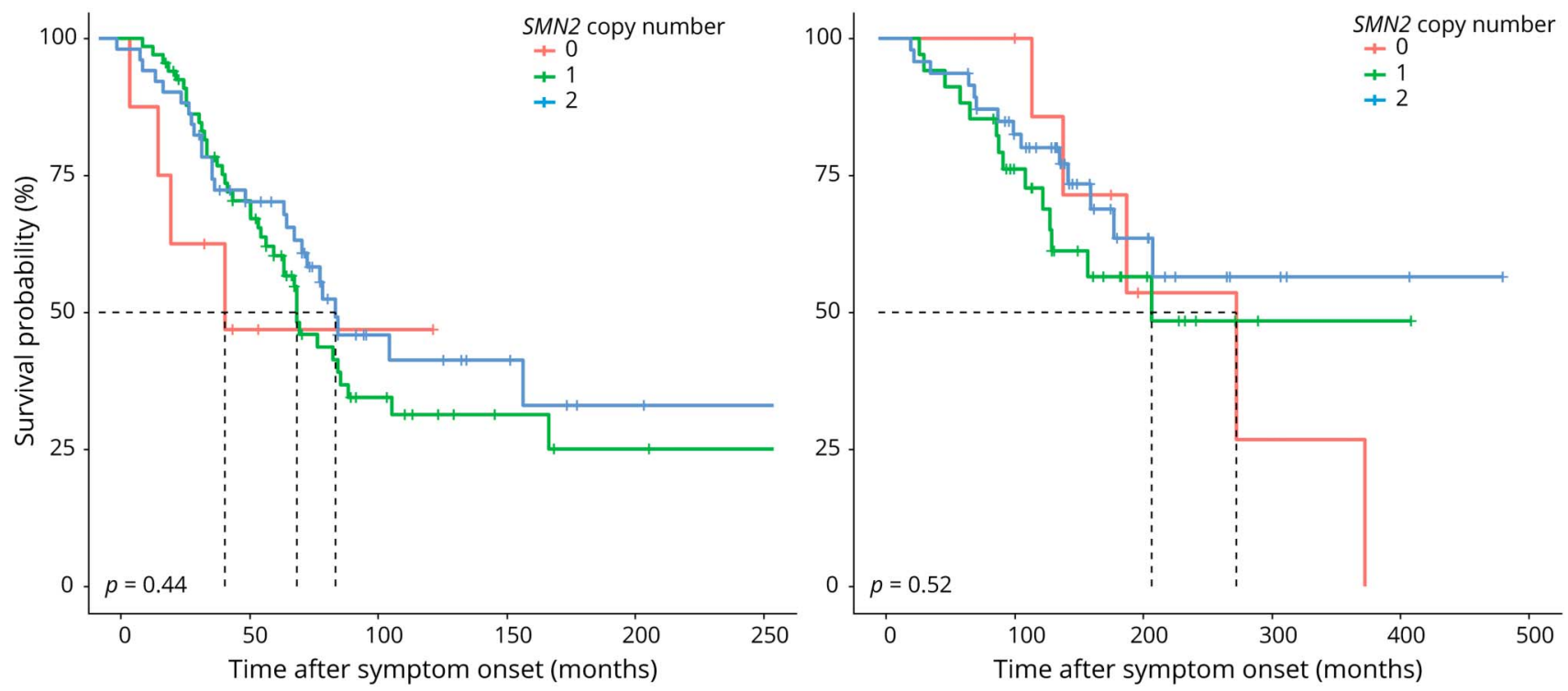

Kaplan-Meier curves showing the probability of survival according to disease duration in patients with PMA (panels A and C) and PLS (panels B and D). Panels A and C show overall survival stratified by the SMN1 copy number. Panels B and D show overall survival stratified by the SMN2 copy number in patients carrying 2 SMN1 copies. Because of low numbers, survival curves for patients with SMN1 deletions and SMN2 duplications are not shown. Survival did not differ between patients with PMA carrying 2 or 3 SMN1 copies (log-rank test $p 0.16$, HR 1.6 [0.83-3.0], $p$ 0.16), nor did the SMN2 copy number have an effect on survival in patients carrying 2 SMN1 copies (log-rank test $p 0.44 ;$ HR 0 vs 2 copies: 1.7 [0.57-4.8], $p$ 0.35; HR 1 vs 2 copies: 1.3 [0.76-2.1], $p$ 0.38). In patients with PLS, the SMN1 copy number was not associated with survival (log-rank test $p 0.22$, HR 0.31 [0.04-2.2], $p$ 0.25), nor did the SMN2 copy number affect survival in patients carrying 2 SMN1 copies (log-rank test $p$ 0.52; HR 0 vs 2 copies: 1.4 [0.51-4.1], $p$ 0.49; HR 1 vs 2 copies: 1.4 [0.69-3.0], $p$ 0.33). HR = hazard ratio; PLS = primary lateral sclerosis; PMA = progressive muscular atrophy; SMN = survival motor neuron.

MMN, PMA, and PLS, and the effect of SMN1 and SMN2 CN on MMN, PMA, and PLS disease course. We found that, compared with controls, SMN1 and SMN2 CN were not associated with MMN and PLS, nor their disease characteristics. PMA, but not its disease course, was associated with SMN1 duplications (OR 2.69 [1.43-4.91], $p$ 0.0020). These results indicate that $\mathrm{CNV}$ in the $S M N$ locus is a novel genetic risk factor for PMA.
Previous studies have shown an association between ALS and SMN1 duplications with a combined OR of 1.76 (95\% CI $1.33-2.32) .^{4,17,18}$ Of interest, a recent study including $>6,000$ patients with ALS identified no association between ALS and SMN CNV. ${ }^{19}$ To better understand these conflicting results and detail the relevance of $\mathrm{CNV}$ in the SMN locus for motor neuron disorders, we have taken a closer look at the extremes of the motor neuron disorder spectrum by analyzing large 
Table 3 Genetic Architecture of SMN1 Duplications in Controls and Patients With PMA

\begin{tabular}{|c|c|c|c|}
\hline & Controls $(n=46)$ & $\operatorname{PMA}(n=15)$ & $p$ Value \\
\hline \multicolumn{4}{|c|}{ [SMN1:SMN2] (n, \%) } \\
\hline $3: 0$ & $6(13)$ & $2(13)$ & 0.64 \\
\hline 3:1 & $20(43)$ & $4(27)$ & \\
\hline $3: 2$ & $16(35)$ & $8(53)$ & \\
\hline $3: 3$ & $4(9)$ & $1(7)$ & \\
\hline \multicolumn{4}{|c|}{ SMN1/2 } \\
\hline 0 & $36(78)$ & $14(93)$ & 0.26 \\
\hline 1 & $10(22)$ & $1(7)$ & \\
\hline \multicolumn{4}{|c|}{ NAIP-5 (n, \%) } \\
\hline 2 & $11(24)$ & $5(33)$ & 0.51 \\
\hline 3 & $31(67)$ & $10(67)$ & \\
\hline 4 & $4(9)$ & $0(0)$ & \\
\hline
\end{tabular}

Abbreviations: NAIP-5 = NLR family apoptosis inhibitory protein exon 5; PMA = progressive muscular atrophy; SMN = survival motor neuron.

cohorts of MNDs characterized by inflammation (MMN), predominant LMN (PMA), and UMN degeneration (PLS). We found an association between SMN1 duplications and PMA, but not PLS and MMN. In PMA, we found a similar effect size identified in a previous study. ${ }^{20}$ We hypothesize that our findings highlight the primary effect of altered $S M N$ $\mathrm{CN}$ on LMNs.

PMA and PLS are considered part of a spectrum of MNDs that also includes ALS. In contrast to ALS and PLS, PMA is limited to clinical signs of LMNs. ${ }^{5}$ However, up to $50 \%$ of patients with PMA may show signs of corticospinal tract degeneration at autopsy. ${ }^{21}$ Whether PMA represents a distinct disease remains a topic of ongoing debate, and further research is warranted to further detail the clinical heterogeneity associated with PMA. We nevertheless think that our PMA cohort differs from a typical ALS cohort on various grounds. Based on detailed clinical and electrophysiologic examination, the patients included in this study showed exclusive progressive loss of LMNs, and almost none of the patients had a bulbar onset. Moreover, in 104 of 159 (65\%) patients, survival was longer than 4 years, and 37 of 159 (23\%) patients had a very long survival of at least 8 years (range 96-531 months). Analysis of available follow-up data of 54 of 159 (34\%) patients showed a conversion to ALS in only 3 cases (i.e., upper motor neuron signs after the start of this study). Of these patients, none carried an SMN1 duplication.

A central question in this study and in the aforementioned studies is what effect SMN1 duplications have on LMN SMN protein levels. In our analysis, we showed that the genetic architecture of SMN1 duplications in PMA does not differ from that of controls, and that about three-quarters of SMN1 duplications are large, including the telomeric NAIP gene. Most importantly, we showed that SMN1 duplications are probably balanced and could theoretically lead to a full-length SMN transcript. Although the transcriptional, translational, and posttranslational effects in individuals carrying 3 SMN1 genes remain unknown, we cannot exclude the possibility that the association between SMN1 duplications and PMA is caused by an excess of LMN SMN protein levels. Further supporting this hypothesis may be the fact that previous studies have not shown an association with SMN1 deletions. ${ }^{4,17,18}$ Alternative explanations may be found in the disruption of regulatory sequences by the large duplications in the SMN locus. Future research on SMN protein levels in, for example, induced pluripotent stem cell-derived motor neurons obtained from patients with different SMN1 genotypes could further address this issue.

To the best of our knowledge, this is the first study reporting on CNV in the SMN locus in MMN and PLS. The size of the cohorts we included in our analyses is substantial, considering the low prevalence of these diseases. Clinically, patients with MMN were characterized in detail, and we had data from longer periods of follow-up for analysis. Our data virtually exclude the $S M N$ locus as a susceptibility locus for inflammatory MNDs. Although our cohort of patients with PMA is also relatively large, it is not possible to draw definite conclusions about the association of SMN CNV and disease course because of a relatively small number of patients with SMN1 duplications. SMN CN varies considerably among different ethnicities. ${ }^{15,16}$ The results of our study therefore cannot readily be extrapolated to ethnicities other than that of the patients in this study, that is, Dutch.

In summary, our study shows that SMN CNV underlies motor neuron vulnerability in PMA, but not in MMN and PLS. This extends the role of SMN CNV in LMN diseases beyond SMA and supports the notion that the SMN locus is a general modifier for LMN disease susceptibility. The intriguing finding that, rather than loss of SMN as in SMA, gene duplications are a main genetic risk factor for PMA warrants further fundamental research to improve our understanding of the cellular mechanisms that underlie this susceptibility.

\section{Acknowledgment}

The authors thank Professor Jan Veldink, MD, PhD, for his help in providing samples for the control cohort.

\section{Study Funding}

The authors report no targeted funding.

\section{Disclosure}

J.W. Bos reports no disclosures. E.J.N. Groen serves on the scientific advisory board for SMA Europe. R.I. Wadman reports no disclosures. C.A.D. Curial reports no disclosures. N.N. Molleman, M. Zegers, P.W.J. van Vught, R. Snetselaar, and R. Vijzelaar are employees of MRC Holland, provider of 
commercially available MLPA assays. W.L. van der Pol serves on the scientific advisory board for SMA Europe, is a member of the Branaplam data monitoring committee (DMC) for Novartis, provides ad hoc consultancy for Biogen and AveXis, and receives research support from the Prinses Beatrix Spierfonds, Vriendenloterij, and Stichting Spieren voor Spieren. L.H. van den Berg serves on the scientific advisory boards for Orion, Biogen, and Cytokinetics; received an educational grant from Takeda; serves on the editorial boards of Amyotrophic Lateral Sclerosis and Frontotemporal Degeneration and the Journal of Neurology, Neurosurgery, and Psychiatry; and receives research support from the Prinses Beatrix Spierfonds, the Netherlands ALS Foundation, and the Netherlands Organization for Health Research and Development (Vici Scheme, JPND [SOPHIA, STRENGTH, ALSCare]). Go to Neurology.org/NG for full disclosures.

\section{Publication History}

Received by Neurology: Genetics January 21, 2021. Accepted in final form April 2, 2021.

Appendix Authors

\begin{tabular}{lll}
\hline Name & Location & Contribution \\
\hline Jeroen W. Bos, MD & $\begin{array}{l}\text { UMC } \\
\text { Utrecht }\end{array}$ & $\begin{array}{l}\text { Design and conceptualization of the } \\
\text { study, acquisition of data, analysis and } \\
\text { interpretation of data, and drafting and } \\
\text { revising the manuscript for intellectual } \\
\text { content }\end{array}$
\end{tabular}

\begin{tabular}{lll}
\hline $\begin{array}{l}\text { Ewout J.N. Groen, } \\
\text { PhD }\end{array}$ & $\begin{array}{l}\text { UMC } \\
\text { Utrecht }\end{array}$ & $\begin{array}{l}\text { Drafting and revising the manuscript for } \\
\text { intellectual content }\end{array}$ \\
\hline $\begin{array}{l}\text { Renske I. Wadman, } \\
\text { MD PhD }\end{array}$ & $\begin{array}{l}\text { UMC } \\
\text { Utrecht }\end{array}$ & $\begin{array}{l}\text { Drafting and revising the manuscript for } \\
\text { intellectual content }\end{array}$ \\
\hline $\begin{array}{l}\text { Chantall A.D. } \\
\text { Curial, BS }\end{array}$ & $\begin{array}{l}\text { UMC } \\
\text { Utrecht }\end{array}$ & Acquisition of data \\
\hline $\begin{array}{l}\text { Naomi N. } \\
\text { Molleman, BS }\end{array}$ & MRC & $\begin{array}{l}\text { Acquisition of data, and analysis and } \\
\text { interpretation of data }\end{array}$ \\
\hline $\begin{array}{l}\text { Marinka Zegers, BS } \\
\text { MRC } \\
\text { Holland }\end{array}$ & $\begin{array}{l}\text { Acquisition of data, and analysis and } \\
\text { interpretation of data }\end{array}$ \\
\hline $\begin{array}{l}\text { Paul W.J. van } \\
\text { Vught, PhD }\end{array}$ & $\begin{array}{l}\text { MRC } \\
\text { Holland }\end{array}$ & $\begin{array}{l}\text { Design and conceptualization } \\
\text { of the study, and drafting and } \\
\text { revising the manuscript for } \\
\text { intellectual content }\end{array}$ \\
\hline $\begin{array}{l}\text { Reinier Snetselaar, } \\
\text { PhD }\end{array}$ & $\begin{array}{l}\text { MRC } \\
\text { Holland }\end{array}$ & $\begin{array}{l}\text { Design and conceptualization } \\
\text { of the study, analysis and } \\
\text { interpretation of data, and } \\
\text { drafting and revising the manuscript } \\
\text { for intellectual content }\end{array}$ \\
\hline
\end{tabular}

Appendix (continued)

\begin{tabular}{|c|c|c|}
\hline Name & Location & Contribution \\
\hline $\begin{array}{l}\text { Raymon Vijzelaar, } \\
\text { PhD }\end{array}$ & $\begin{array}{l}\text { MRC } \\
\text { Holland }\end{array}$ & $\begin{array}{l}\text { Design and conceptualization of the } \\
\text { study, analysis and interpretation of data, } \\
\text { and drafting and revising the manuscript } \\
\text { for intellectual content }\end{array}$ \\
\hline $\begin{array}{l}\text { W. Ludo van der } \\
\text { Pol, MD, PhD }\end{array}$ & $\begin{array}{l}\text { UMC } \\
\text { Utrecht }\end{array}$ & $\begin{array}{l}\text { Design and conceptualization of the } \\
\text { study, and drafting and revising the } \\
\text { manuscript for intellectual content }\end{array}$ \\
\hline $\begin{array}{l}\text { Leonard H. van den } \\
\text { Berg, MD, PhD }\end{array}$ & $\begin{array}{l}\text { UMC } \\
\text { Utrecht }\end{array}$ & $\begin{array}{l}\text { Design and conceptualization of the } \\
\text { study, and drafting and revising the } \\
\text { manuscript for intellectual content }\end{array}$ \\
\hline
\end{tabular}

\section{References}

1. Butchbach MER. Copy number variations in the Survival Motor Neuron genes: implications for spinal muscular atrophy and other neurodegenerative diseases. Front Mol Biosci. 2016:3:7.

2. Wadman RI, Jansen MD, Stam M, et al. Intragenic and structural variation in the SMN locus and clinical variability in spinal muscular atrophy. Brain Commun. 2020;2(2):fcaa075.

3. Wirth B, Karakaya M, Kye MJ, Mendoza-Ferreira N. Twenty-five years of spinal muscular atrophy research: from phenotype to genotype to therapy, and what comes next. Annu Rev Genomics Hum Genet. 2020;21:231-261.

4. Wang X, Cui N, Gao J, Qiu X, Zheng F. SMN1 duplications contribute to sporadic amyotrophic lateral sclerosis susceptibility: evidence from a meta-analysis. J Neurol Sci. 2014;340(1-2):63-68.

5. Van Es MA, Hardiman O, Chio A, et al.. Amyotrophic lateral sclerosis. Lancet. 2017; 390(10107):2084-2098.

6. Vlam L, van der Pol WL, Cats EA, et al. Multifocal motor neuropathy: diagnosis, pathogenesis and treatment strategies. Nat Rev Neurol. 2011;8(1):48-58.

7. Harschnitz O, Jongbloed BA, Franssen H, Straver DC, van der Pol WL, van den Berg LH. MMN: from immunological cross-talk to conduction block. J Clin Immunol. 2014;34(suppl 1):S112-S119.

8. Herraets I, Van Rosmalen M, Bos J, et al. Clinical outcomes in multifocal motor neuropathy: a combined cross-sectional and follow-up study. Neurology. 2020;95(14):1979-1987.

9. Sutedja NA, Otten HG, Cats EA, et al. Increased frequency of HLA-DRB1*15 in patients with multifocal motor neuropathy. Neurology. 2010;74(10):828-832.

10. Liewluck T, Saperstein DS. Progressive muscular atrophy. Neurol Clin. 2015;33(4):761-773.

11. van Schaik IN, Leger JM, Nobile-Orazio E, et al. European Federation of Neurological Societies/Peripheral Nerve Society guideline on management of multifocal motor neuropathy. Report of a joint task force of the European Federation of Neurological Societies and the Peripheral Nerve Society-first revision. J Peripher Nerv Syst. 2010; 15(4):295-301.

12. Huisman MHB, Seelen M, De Jong SW, et al. Lifetime physical activity and the risk of amyotrophic lateral sclerosis. J Neurol Neurosurg Psychiatry 2013;84(9):976-981s.

13. Cats EA, van der Pol WL, Piepers S, et al. Correlates of outcome and response to IVIg in 88 patients with multifocal motor neuropathy. Neurology. 2010;75(9):818-825.

14. Cats EA, Jacobs BC, Yuki N, et al. Multifocal motor neuropathy: association of antiGM1 IgM antibodies with clinical features. Neurology. 2010;75(22):1961-1967.

15. Vijzelaar R, Snetselaar R, Clausen M, et al. The frequency of SMN gene variants lacking exon 7 and 8 is highly population dependent. PLoS One. 2019;14(7):e0220211.

16. Vorster E, Essop FB, Rodda JL, Krause A. Spinal muscular atrophy in the black South African population: a matter of rearrangement? Front Genet. 2020;11(54).

17. Blauw HM, Barnes CP, Van Vught PWJ, et al. SMN1 gene duplications are associated with sporadic ALS. Neurology. 2012;78(11):776-780.

18. Veldink JH, Kalmijn A, Van der Hout AH, et al. SMN genotypes producing less SMN protein increase susceptibility to and severity of sporadic ALS. Neurology. 2005;65(6):820-825.

19. Moisse M, Zwamborn RAJ, Van Vugt J, et al. The effect of SMN gene dosage on ALS risk and disease severity. Ann Neurol. 2021;89(4):686-697.

20. Kuźma-Kozakiewicz M, Jȩdrzejowska M, Kaźmierczak B. SMN1 gene duplications are more frequent in patients with progressive muscular atrophy. Amyotroph Lateral Scler Frontotemporal Degener. 2013;14(5-6):457-462.

21. Ince PG, Evans J, Knopp M, et al. Corticospinal tract degeneration in the progressive muscular atrophy variant of ALS. Neurology. 2003;60(8):1252-1258. 


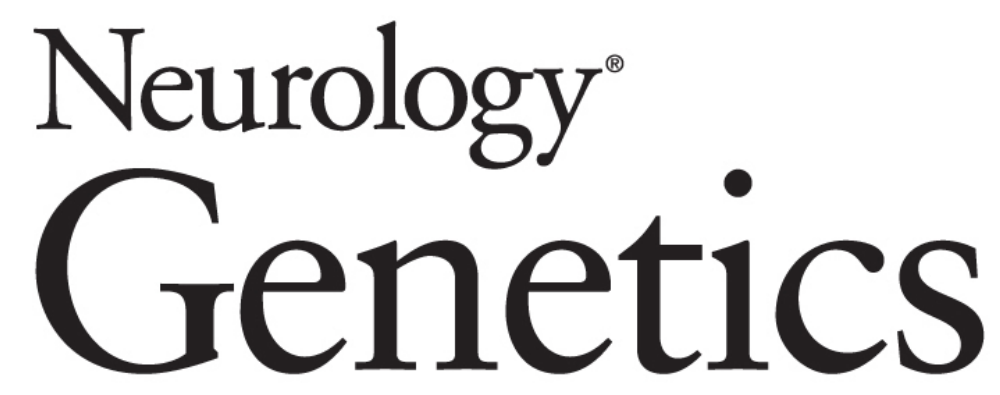
SMN1 Duplications Are Associated With Progressive Muscular Atrophy, but Not With Multifocal Motor Neuropathy and Primary Lateral Sclerosis
Jeroen W. Bos, Ewout J.N. Groen, Renske I. Wadman, et al. Neurol Genet 2021;7;
DOI 10.1212/NXG.0000000000000598

This information is current as of June 22, 2021

\begin{abstract}
Updated Information \& Services

References

Subspecialty Collections

Permissions \& Licensing

Reprints

including high resolution figures, can be found at: http://ng.neurology.org/content/7/4/e598.full.html

This article cites 20 articles, 1 of which you can access for free at: http://ng.neurology.org/content/7/4/e598.full.html\#\#ref-list-1

This article, along with others on similar topics, appears in the following collection(s):

Association studies in genetics

http://ng.neurology.org//cgi/collection/association_studies_in_genetics

Information about reproducing this article in parts (figures,tables) or in its entirety can be found online at:

http://ng.neurology.org/misc/about.xhtml\#permissions

Information about ordering reprints can be found online: http://ng.neurology.org/misc/addir.xhtml\#reprintsus
\end{abstract}

Neurol Genet is an official journal of the American Academy of Neurology. Published since April 2015, it is an open-access, online-only, continuous publication journal. Copyright Copyright $\odot 2021$ The Author(s). Published by Wolters Kluwer Health, Inc. on behalf of the American Academy of Neurology.. All rights reserved. Online ISSN: 2376-7839.

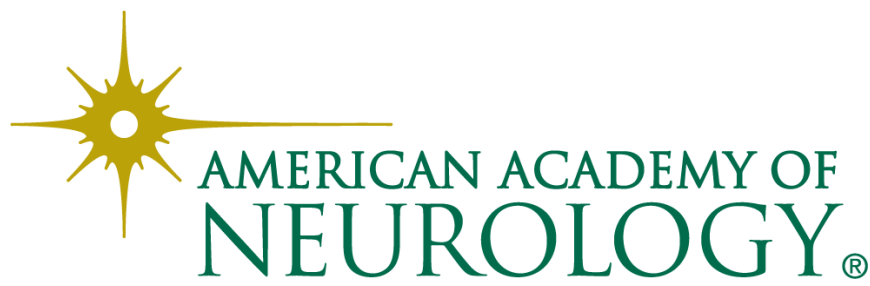

